\title{
Confinement temporel de la génération d'harmoniques d'ordre élevé
}

\author{
O. Tcherbakoff, J. Plumridge, D. Descamps, E. Mével, F. Salin et E. Constant
}

\author{
CELIA, UMR 5107 du CNRS, Université Bordeaux I, 351 cours de la Libération, \\ 33405 Talence cedex, France
}

\begin{abstract}
Résumé : On utilise l'extrême sensibilité des harmoniques à l'ellipticité du champ laser fondamental pour confiner temporellement l'émission harmonique. Nous utilisons une technique linéaire permettant de moduler l'état de polarisation du champ laser infra-rouge de durée $\tau=35 \mathrm{fs}$. On créé ainsi dans l'impulsion laser une porte temporelle où la polarisation est linéaire, et est elliptique en dehors. La porte temporelle de polarisation linéaire est ajustable continûment d'une valeur minimum de 7 fs à l'infini. On génère l'émission harmonique avec cette impulsion laser modulée en polarisation pour observer l'évolution du spectre harmonique en fonction de la durée de la porte temporelle. Les spectres observées expérimentalement sont en bon accord avec un confinement temporelle en deçà de $7 \mathrm{fs}$. Avec un champ laser fondamental plus court, on devrait être capable d'isoler une seule impulsion attoseconde.
\end{abstract}

\section{INTRODUCTION}

Les harmoniques d'ordre élevé, produites lors de l'interaction entre un laser impulsionnel intense et un milieu gazeux, sont émises dans le domaine temporel sous forme d'un train d'impulsions attosecondes espacées d'une demi période optique du champ laser [1]. La production d'une seule impulsion attoseconde permettrait d'accéder à des résolutions temporelles jusqu'ici inaccessibles pour des études résolues en temps. D'après les simulations [2,3,4], une des solutions pour atteindre cet objectif est de confiner la génération d'harmoniques sur moins d'un cycle optique du laser fondamental.

Dans cet article, nous présentons une méthode qui permettrait de restreindre l'émission harmonique à une seule impulsion. Elle repose sur l'extrême sensibilité de la génération d'harmoniques avec la polarisation et l'intensité du laser incident [5,6]. Nous avons testé une technique [7] permettant de moduler temporellement la polarisation d'une impulsion laser infra-rouge de durée $\tau \cdot 35 \mathrm{fs}$. On crée, ainsi, dans l'impulsion résultante une porte temporelle où la polarisation est quasiment linéaire pendant environ $7 \mathrm{fs}$, ce qui correspond à 5 impulsions attosecondes dans le train XUV. Pour observer les effets du confinement sur la génération d'harmonique, nous étudions les modifications spectrales des harmoniques situées dans le plateau et dans la coupure.

\section{DISPOSITIF EXPÉRIMENTAL}

\subsection{Génération d'harmonique}

Nous utilisons la chaîne laser du CELIA délivrant à $800 \mathrm{~nm}$ des impulsions de durée 35 fs et une énergie atteignant $10 \mathrm{~mJ}$ avec un taux de répétition de $1 \mathrm{kHz}$. La manipulation de la polarisation de l'impulsion laser, se réalise via deux lames biréfringentes : une lame quart d'onde d'ordre multiple d'épaisseur 1.04 $\mathrm{mm}$ et une lame également quart d'onde d'ordre 0 . Le faisceau est focalisé à l'aide d'une lentille de $1.5 \mathrm{~m}$ 
dans une cellule de longueur $1.5 \mathrm{~cm}$ et de diamètre $400 \mu \mathrm{m}$ remplie d'argon. Le spectre harmonique est analysé à l'aide d'un spectromètre XUV comportant un miroir torique en incidence rasante et un réseau plan puis détecté à l'aide d'une caméra $\mathrm{CCD}$, protégée par un filtre en aluminium d'épaisseur $1000 \AA$ monté sur une grille (Fig 1).

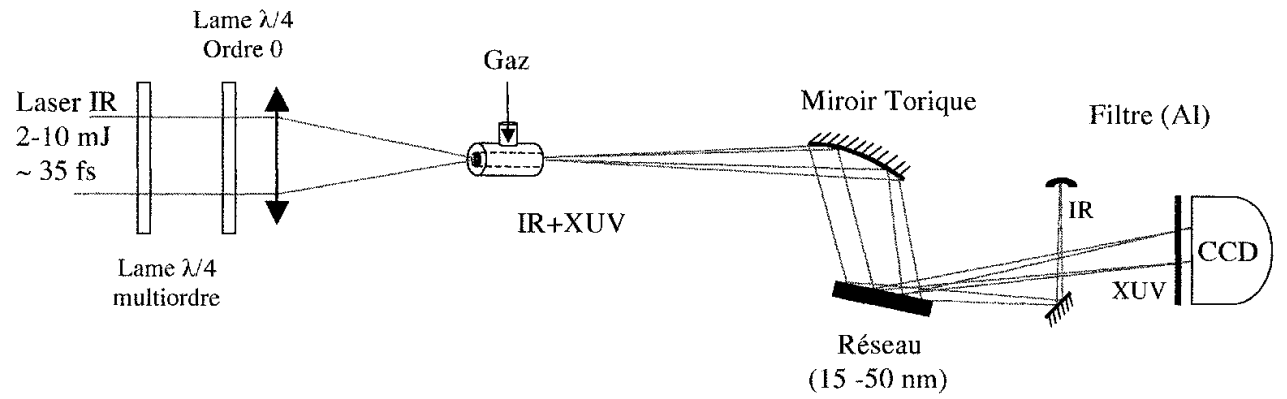

Figure 1 : Dispositif expérimental

\subsection{Modulation de l'ellipticité de l'impulsion infra-rouge}

Une impulsion polarisée à $45^{\circ}$ des axes neutres de la première lame est transformée en deux impulsions d'égales amplitudes, de polarisations linéaires croisées et décalées de $32 \mathrm{fs}$. Le profil de l'impulsion résultante s'assimile alors un profil carré de durée $\approx 64 \mathrm{fs}$. En raison du déphasage, multiple de $\pi / 2$, la polarisation est linéaire en début et fin d'impulsion et varie continûment jusqu'à devenir circulaire au centre de l'impulsion. L'impulsion traverse ensuite la seconde lame dont les axes neutres sont tournés d'un angle $\beta$ par rapport aux axes de la première lame. Pour $\beta=45^{\circ}$, la polarisation linéaire devient circulaire et vice versa, pour $\beta=0$, la polarisation est linéaire tout le long de l'impulsion.

Pour les harmoniques 17 à 29 qui ont été étudiées, l'efficacité est maximale en polarisation linéaire et diminue de $50 \%$ pour une ellipiticitéé de $13 \%$. On utilise ce critère pour définir la largeur de la porte temporelle pendant laquelle l'ellipiticité reste inférieure à $13 \%$. Elle est égale à $\tau / 5$ soit à peu près $7 \mathrm{fs}$. En tournant la $2^{\text {nd }}$ lame d'un angle $\beta$, on varie continûment la largeur de la porte temporelle de polarisation depuis 7 fs $\left(\beta=45^{\circ}\right.$, porte étroite) jusqu'à l'infini $\left(\beta=0^{\circ}\right.$, porte large) et ce en préservant le profil d'intensité de l'impulsion (Fig 2).

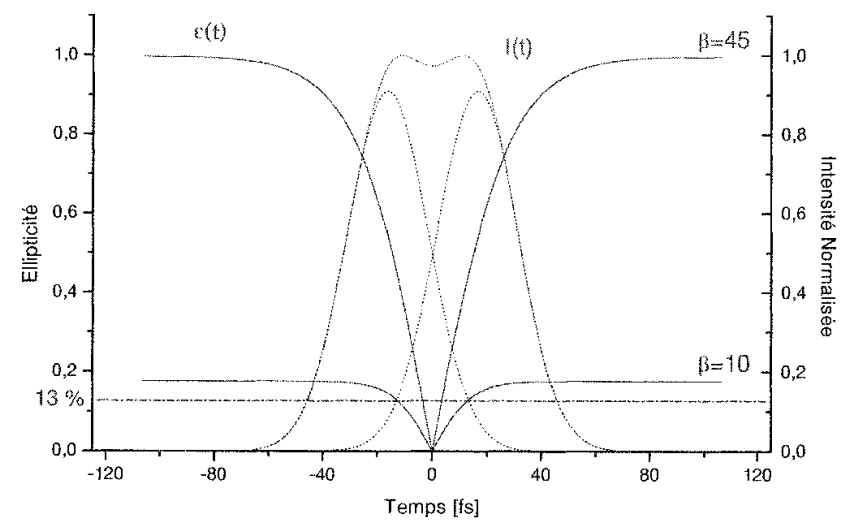

Figure 2 : Profils temporels d'intensité et d'ellipticité après traversée des deux lames $\lambda / 4$.

(....) profils des deux demi impulsions après la traversée de la première lame. (---) profil d'intensité résultant. (-

l'ellipticité de l'impulsion pour deux angles de la lame quart d'onde d'ordre 0 différents $\beta=45$ (porte étroite) et $\beta=10$.

$1 \varepsilon=100 \%$ polarisation circulaire, $\varepsilon=0 \%$ polarisation linéaire 


\section{RÉSULTATS EXPÉRIMENTAUX}

\subsection{Spectre harmonique}

Nous avons étudié l'évolution de la largeur spectrale des harmoniques en fonction de la durée de la porte temporelle. Les résultats obtenus pour les harmoniques du plateau sont assez contre intuitifs. En confinant la génération d'harmonique à un temps très court, la largeur spectrale des harmoniques du plateau décroît (vu dans [8]) (Fig 3-a ). Par contre, pour les harmoniques de la coupure, on observe un élargissement de la largeur spectrale lorsque la durée temporelle de la porte décroît (Fig 3-b), résultat auquel on s'attend. Pour expliquer ces comportements opposés il faut tenir compte à la fois de la dépendance en intensité de la phase induite par le dipôle atomique et du confinement de la génération d'harmonique.

Un modèle théorique basé sur une formulation quantique permet de décrire la génération d'harmoniques $[9,10]$. Pour les harmoniques du plateau, ce modèle montre que la phase induite par le dipôle est associée à l'action des deux trajectoires électroniques prédominantes dans la production d'harmonique. La trajectoire $\tau_{1}$ correspond à un temps d'oscillation dans le continuum proche de la demi période optique tandis que le temps associé à la trajectoire $\tau_{2}$ est inférieur à la période optique. La contribution de la phase du chemin quantique $\tau_{1}$ varie linéairement et lentement avec l'intensité du laser alors que la contribution de la phase du chemin quantique $\tau_{2}$ est plus importante. En tenant compte de la contribution des différentes trajectoires électroniques, le champ harmonique à une fréquence $\omega_{\mathrm{q}} \mathrm{s}$ 'écrit sous la forme de deux composantes $i=1,2$ avec une dépendance en phase de la forme:

$$
\phi_{i}=-\alpha_{\mathrm{i}} \mathrm{I}(\mathrm{r}, \mathrm{z}, \mathrm{t}) \text { avec } \alpha_{1}=1 \times 10^{14} \mathrm{~cm}^{2} / \mathrm{W} \text { et } \alpha_{2}=27 \times 10^{\mathrm{i} 4} \mathrm{~cm}^{2} / \mathrm{W}
$$

La formulation est identique pour les harmoniques de la coupure à la différence près qu'il existe une seule trajectoire dominante donc une seule composante pour le champ harmonique dont la dépendance en phase s'écrit comme (1) avec $\alpha=12 \times 10^{14} \mathrm{~cm}^{2} / \mathrm{W}$.

Sans le confinement, les harmoniques du plateau sont principalement générées pendant le front montant de l'impulsion. La variation d'intensité entraîne une modulation de la phase, et par conséquent un élargissement spectral des harmoniques. Les harmoniques de la coupure sont générées au sommet de l'impulsion où l'intensité est constante, l'élargissement spectral du à la modulation de phase est beaucoup moins important pour les harmoniques de la coupure que pour à celles du plateau.

Le confinement à deux effets opposés : la largeur spectrale a tendance à diminuer puisque la variation d'intensité est quasi nulle là où l'impulsion est polarisée linéairement, et par conséquent la modulation de la phase est pratiquement nulle. La réduction de l'émission harmonique à quelques cycles optiques a tendance à élargir le spectre harmonique. C'est la compétition entre ces deux phénomènes qui donne soit un élargissement, soit un rétrécissement du spectre harmonique. Dans le cas des harmoniques du plateau, l'influence de la phase atomique est dominante, c'est un rétrécissement avec en outre un décalage spectral vers les basses fréquences. Pour les harmoniques de la coupure, l'effet du confinement temporel est dominant, ce qui se traduit par un élargissement spectral.

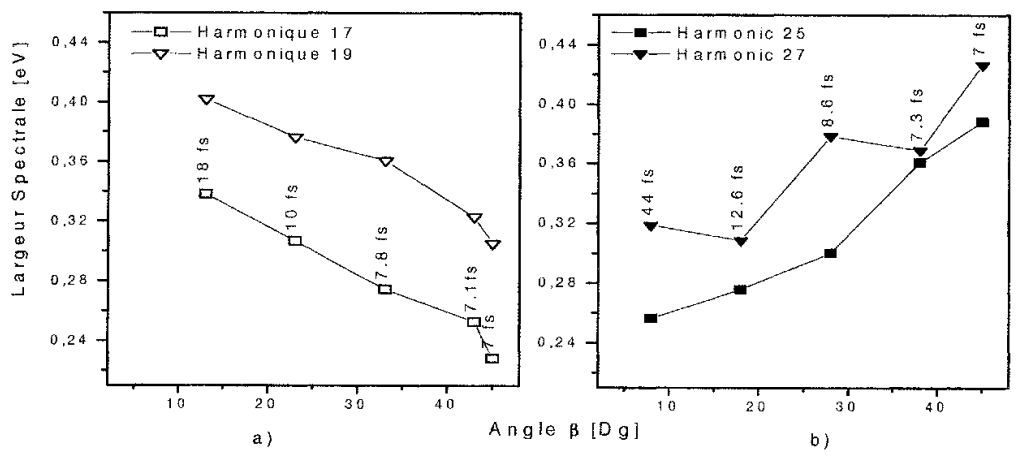

Figure 3: a) Largeur spectrale des harmoniques 17 (- $\square$-) et $19(-\nabla-)$ (plateau). b) Largeur spectrale des harmoniques 25 (-) et $27\left(-\boldsymbol{\nabla}_{-}\right)$(coupure).en fonction de la durée de la porte temporelle. Énergie $1,3 \mathrm{~mJ}$, pression dans la cellule $10 \mathrm{mbar}$.

Pour exploiter ces résultats il faut minimiser les autres causes éventuelles d'élargissement, il faut s'affranchir le plus possible des effets dus à l'ionisation du gaz et à la modulation de phase du faisceau 
infrarouge. Pour ce faire, nous nous sommes limités à de faibles énergies et basses pressions dans la cellule. De plus, pour minimiser les effets de la modulation de phase du fondamental, l'impulsion infrarouge doit être proche de sa limite de Fourier ou en d'autre termes avoir une durée la plus courte, ce réglage est effectué à l'aide du compresseur de la chaîne laser en optimisant le signal à $2 \omega$ (directement proportionnel à la durée de l'impulsion) issu d'un cristal de BBO placé après la cellule de gaz.

On estime la largeur de l'harmonique due au confinement en considérant que les différentes largeurs spectrales sont décorrélées on écrit alors :

$$
\Delta \mathrm{E}_{\mathrm{pe}}=\sqrt{\Delta \mathrm{E}_{\mathrm{pl}}{ }^{2}+\Delta \mathrm{E}_{\mathrm{conf}}{ }^{2}}
$$

Avec $\Delta \mathrm{E}_{\mathrm{pe}}$ : largeur spectrale de l'harmonique avec la porte étroite, $\Delta \mathrm{E}_{\mathrm{pl}}$ : largeur spectrale de l'harmonique avec la porte large, $\Delta \mathrm{E}_{\text {conf }}$ : largeur spectrale confinée de l'harmonique. En considérant l'harmonique 27, on a de $\Delta \mathrm{E}_{\mathrm{pl}}=0,32 \mathrm{eV}, \Delta \mathrm{E}_{\mathrm{pe}}=0,42 \mathrm{eV}$. La largeur due au confinement est $\Delta \mathrm{E}_{\mathrm{conf}}=0,27$ $\mathrm{eV}$. Un profil d'intensité gaussien limité par sa transformée de Fourier a sa largeur spectrale et sa largeur temporelle liées par la relation suivante $\Delta \mathrm{E}_{(\mathrm{cV})} \times \Delta \mathrm{t}_{\mathrm{t( \textrm {s } )}}=1,8$. Pour $\Delta \mathrm{E}_{\mathrm{conf}}=0,27 \mathrm{eV}$ on obtient $\Delta \mathrm{t}=6,6 \mathrm{fs}$, cette valeur est consistante avec la durée de la porte étroite créée.

\subsection{Efficacité de la génération d'harmonique}

L'étude sur l'efficacité de l'émission harmonique en fonction de l'énergie incidente pour la porte étroite et la porte large, montre des comportements assez surprenants. Sous certaines conditions et principalement pour les harmoniques de la coupure on arrive à générer plus d'harmonique avec la porte étroite que la porte large. Nous attribuons ces effets aux conditions d'accord de phase et de volume, néanmoins nous n'avons pas poussé l'étude plus en avant.

\section{CONCLUSION}

L'observation simultanée d'un élargissement pour les harmoniques de la coupure et un rétrécissement pour les harmoniques du plateau par confinement temporel, est une première étape prometteuse. Les spectres obtenus sont bien compatibles avec un confinement de la génération d'harmonique à $\approx 7 \mathrm{fs}$. En utilisant une impulsion de durée $15 \mathrm{fs}$, il est envisageable de confiner la génération d'harmonique à moins d'une période optique et par conséquent de générer une seule impulsion attoseconde. La caractérisation temporelle de cette impulsion pourrait se réaliser à l'aide d'un autocorrélateur XUV dont la mise au point est en train de s'effectuer dans le laboratoire.

\section{Références}

[1] P.M. Paul et al Science 292, (2001), 1689

[2] P.B Corkum et al Opt. Lett. 19, (1994),1870

[3] V.T. Platonenko et al Opt. Soc Am. B., 16, (1999), 435

[4] Ph. Antoine et al Phys Rev A 56, (1997),4960

[5] K.S. Budil et al Phys Rev A 48, (1993), 3437

[6] Ph. Antoine et al Phys. Rev. A, 53, (1996), 1725

[7] E. Constant Thèse de Doctorat (1997)

[8] C.Altucci et al Phys. Rev. A, 58, (1998), 3934

[9] M. Lewenstein et al Phys. Rev. A, 52, (1995),4747,

[10] M. Lewenstein et al Phys. Rev. A, 49, (1994),2117 